\title{
HIGH STRAIN AND BIOCOMPATIBLE SCREEN PRINTED NANOCOMPOSITE BASED CONDUCTIVE PDMS STRAIN SENSORS
}

\author{
C. Lee, L. Jug and E. Meng \\ University of Southern California, Los Angeles, California, USC
}

\begin{abstract}
Strain sensors capable of operating in high strain conditions remain a technical challenge. In particular, biocompatible strain sensor technology is needed that satisfies the following requirements: (1) construction from low modulus materials that approach values of soft biological tissues and (2) high strain operation ( $\geq 20 \%)$. We present a screen printed, nanocomposite-based conductive polydimethylsiloxane (CPDMS) strain sensor capable of $40 \%$ strain operation with a gauge factor (GF) $>100$. Strain sensing using CPDMS sensors containing multi-walled carbon nanotubes (MWNT), graphene nanoplatelets (GNP), or a mixture of both nanocarbon filler materials was demonstrated. The combination of high strain operation, high GF, and biocompatible construction pave the way for minimally invasive in vivo strain measurements. Strain sensors were characterized according to their conductivity, zero current resistance (ZCR), thermal coefficients of resistance (TCR), and gauge factor.
\end{abstract}

\section{INTRODUCTION}

Typical strain sensors are made from silicon, metal, or other hard materials using microelectromechanical systems (MEMS) technology [1-2]. These devices are efficient strain sensors; however, they are limited to low strain applications, and their modulus of elasticity does not match that of soft tissues [3], thus limiting their ability to be used in vivo for measuring the strain of soft tissues. Recent research has shown that polydimethylsiloxane mixed with nanocarbon filler (typically multi-walled carbon nanotubes) has potential as a piezoresistive material for strain gauge applications [4-8] but only low gauge factors $(\sim 12)$ were achieved [9]. Such composites become conductive once the filler concentration reaches the percolation threshold. The percolation threshold of CNTs is dependent on aspect ratio, diameter, degree of conglomeration and alignment and therefore ranges widely from $0.005 \mathrm{vol} \%$ to several vol $\%$ [10].

We investigated a GNP/MWNT blend mixed with PDMS to achieve a low percolation threshold with MWNTs while conductive networks above the percolation threshold were created using GNPs. GNPs are also lower in cost and improve the consistency of the prepolymer for screenprinting. The GNP/MWNT and PDMS composite form CPDMS that is sandwiched between and supported by two layers of transparent, medical grade PDMS.

A medical grade PDMS (USP Class VI and ISO 10993-1) was selected for the polymer matrix to improve elongation properties of the resulting CPDMS for high strain operation, which, in our past work, was limited by

9780964002494/HH2012/\$25C2012TRF fracture of the piezoresistive material under strain (failed at $1.5 \%$ strain [11]). PDMS-based strain sensors are low cost, simple to manufacture, and well suited for in vivo use because of their low Young's modulus (closer match to that of organs and tissues than silicon) and biocompatibility. Our intended application is in vivo measurement of urinary bladder fullness which requires reliable operation up to $\sim 20 \%$ strain.

\section{THEORY}

Gauge factor is a useful measure of merit for strain sensors and is equal to the normalized change in resistance divided by the strain:

$$
G F=\frac{\Delta R / R}{\Delta L / L}=\frac{\Delta R / R}{\varepsilon}
$$

where $R$ is the nominal as-fabricated, undeformed strain gauge resistance, $\Delta R$ is the total change in resistance, $L$ is the original unstrained strain gauge length, $\Delta L$ is the change in length following application of strain, and $\varepsilon$ is the applied strain. Metal and semiconductors are typical materials used for strain gauges. However, metal strain gauges typically have relatively low gauge factors $(\mathrm{GF} \sim 2)$ and although semiconductors have much better gauge factors (GF 100), they can only be used for low strain $(<$ $0.1 \%$ ) applications. Thick film materials for strain sensing have reported GFs in the range of 2-10 [12-14] which are promising for high strain operation applications such as measurement of strain/fullness of the bladder as part of a neuroprosthetic approach to management of lower urinary tract dysfunction [11].

\section{METHODS}

\section{Materials}

Graphene nanoplatelets and multiwalled carbon nanotubes were obtained from cheaptubes.com (Brattleboro, VT), PDMS from Factor II (A-103, MDX44210, Lakeside, AZ), Stoddard solvent and isopropyl alcohol (IPA) from VWR (Brisbane, CA), and 34 gauge wire from EIS/Fay Wire (Elmhurst, IL).

\section{Sensor Fabrication}

To prepare the CPDMS prepolymer mixture, a precision scale was used to measure out a predetermined amount of carbon filler material, either graphene nanoplatelets (GNP) or multiwalled carbon nanotubes (MWNT), and PDMS base into a glass beaker. To this was added $10-15 \mathrm{~mL}$ of a solvent mixture consisting of a 30:70 ratio of IPA and Stoddard solvent (by volume).
Solid-State Sensors, Actuators, and Microsystems Workshop Hilton Head Island, South Carolina, June 3-7, 2012 
The mixture was then placed in an ultrasonic bath (Bransonic 3510, Branson Ultrasonic Corp., Danbury, CT) for 15-18 hours to achieve thorough mixing of the filler material with the PDMS base and evaporate organic solvent. Using this method, CPDMS base with a range of concentrations of MWNT and GNP were prepared (Figure 1).

a

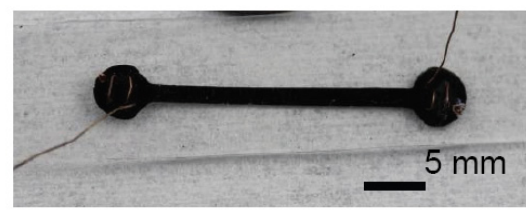

b

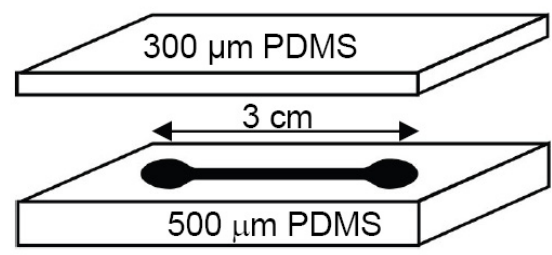

C

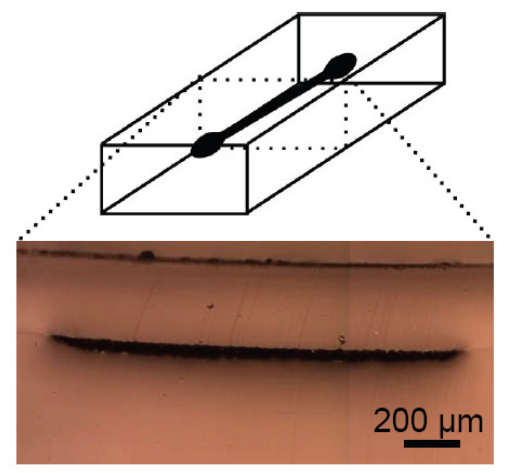

Figure 1: (a) Optical micrograph, (b) three dimensional exploded illustration showing sensor construction, and (c) illustration and optical micrograph showing cross section of strain sensor. (Dimensions of top surface: $9.5 \mathrm{~mm} \times 40 \mathrm{~mm}$.)

Crosslinker (in a ratio of 1:10 to the base) was added to the nanocomposite conductive filler-PDMS base mixture using a planetary mixer (Thinky Corp., Laguna Hills, CA) and the obtained conductive prepolymer was screen printed onto a $500 \mu \mathrm{m}$ thick layer of nonconductive medical grade PDMS using a lithographically defined brass screen using a previously developed method [11]. Briefly, CPDMS prepolymer was spread across a custom stencil using a plastic squeegee. The stencil was made by etching away a lithographically defined pattern in a $76 \mu \mathrm{m}$ thick brass shim $(4.5 " \times 6 ")$ sandwiched between two sheets of a negative, dry-film photoresist. The photoresist was exposed using a high resolution transparency mask (Mikacolor, Los Angeles, CA) and UV light source $\left(45 \mathrm{~mJ} / \mathrm{cm}^{2}\right)$ and developed in a dilute sodium hydroxide bath. The exposed brass regions were etched away using ferric chloride. The parts for this etching kit were purchased from MicroMark (Berkeley Heights, NJ).
The screen-printed CPDMS was then placed under a vacuum for 3-4 hours to remove any remaining solvent and then cured at $80^{\circ} \mathrm{C}$. A second layer of nonconductive medical grade PDMS was added to fully encapsulate the CPDMS. Robust electrical connections were made by threading fine wires through the contact pads several times. For the purposes of saline soak testing, an additional layer of PDMS was added to insulate the threaded wires.

\section{Electrical Properties}

Zero current resistance (ZCR) was measured by recording the resistances of the devices from $1-10 \mu \mathrm{A}$ and extrapolating the resistances back to zero current.

Temperature coefficient of resistance (TCR) was calculated by measuring the device resistance at different temperatures from room temperature to $90^{\circ} \mathrm{C}$ and taking the slope of the resulting curve.

Gauge factor was obtained from data acquired using a motorized stage (Thorlabs, Z812) that allowed for user defined strain patterns and a precision multimeter (Keithley 2400, Keithley Instruments, Cleveland, OH). The sensors were mounted to the motorized stage using custom acrylic clamps with a known separation distance (Figure 2). Clamp separation was increased by discrete steps via computer control of the stage to achieve known levels of unaxial strain. Sensor performance under various strain regimens was evaluated.

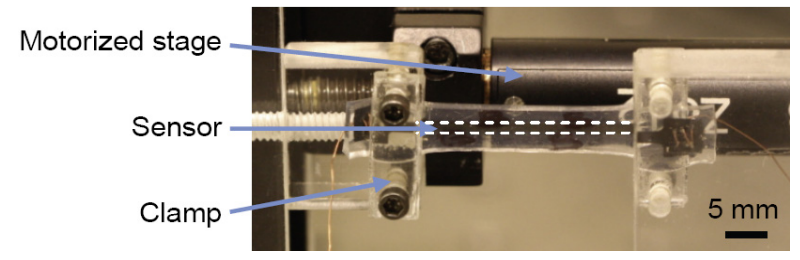

Figure 2: Gauge factor test set up.

\section{RESULTS AND DISCUSSION}

CPDMS mixtures were made with concentrations of MWNT from $2.5 \mathrm{wt} \%$ to $15 \%$. Under $5 \%$, the devices were not conductive and over $10 \%$, the mixture was too viscous to be screen-printed. GNP concentrations were varied from $5 \%$ to $15 \%$. Under $10 \%$, the GNP devices were not conductive and over $12.5 \%$, the CPDMS would not cure. Based on these observations, mixtures of GNP and MWNT were selected ranging from 2.5 to $7.5 \%$ MWNT and 1.5 to $15 \%$ GNP (Table 1).

\section{Electrical Characteristics}

For electrical characterization of the CPDMS material, $35 \times 45 \mathrm{~mm}$ rectangular patterns were printed onto a non-conductive PDMS substrate to ensure that the edges effects were minimized. However, subsequent 4 point probe measurements performed directly on unencapsulated sensors $(1.8 \times 30 \mathrm{~mm})$ yielded identical resistance values, therefore 4 point measurements were 
made directly on exposed, unencapsulated sensors. The thickness of the film was measured by taking sections through a screen-printed sensor element and measuring the average thickness using NIH ImageJ software (Figure 1c).

Table 1. Gauge factor and other manufacturing notes describing different compositions $(N C=$ not conductive. Chloroform was used as a non-polar solvent until it was determined that it is not a suitable solvent for MDX-4 4210. All devices, therefore, were produced using Stoddard solvent and IPA mixture unless otherwise noted.).

\begin{tabular}{|c|c|c|l|}
\hline \multicolumn{2}{|c|}{ Composition } & \multirow{2}{*}{ GF } & \multicolumn{2}{|}{ Notes } \\
\cline { 1 - 2 } MWNT (\%) & GNP (\%) & & \\
\hline 3.5 & 0 & NA & NC \\
\hline 5 & 0 & 99.8 & \\
\hline 10 & 0 & 10.24 & \\
\hline 15 & 0 & NA & Not printable \\
\hline 0 & 5 & NA & NC \\
\hline 0 & 10 & NA & NC \\
\hline 0 & 12.5 & 17.25 & \\
\hline 3.5 & 1.5 & 88.75 & \\
\hline 5 & 10 & 1.18 & Did not cure, chloroform \\
\hline 7.5 & 5 & 2.7 & Chloroform \\
\hline 2.5 & 15 & 2.6 & Did not cure, chloroform \\
\hline 2.5 & 15 & 20.5 & $\begin{array}{l}\text { Did not cure, chloroform, } \\
\text { Parylene coated }\end{array}$ \\
\hline
\end{tabular}

The measured conductivity of the CPDMS was similar to the results of other studies [5-6] (Figure 3), with MWNT devices becoming conductive at $5 \mathrm{wt} \%$. An upper limit to conductivity was found with GNP at $12.5 \%$, which was as conductive as the MWNT samples reported in [5]. Therefore, although conductivity could not be achieved at low concentrations of GNP below $12.5 \%$, once GNP loaded samples became conductive (reached the percolation threshold), they were as conductive of their MWNT counterparts. This trend also appeared when measuring zero current resistance. Although the resistance of the 10\% GNP sample was much higher than the $10 \%$ MWNT sample, when the concentration of GNP was increased to $12.5 \%$, the resistance dropped down to below the $10 \%$ MWNT resistance (Figure 4).

Pure MWNT devices exhibited negative TCR, whereas pure GNP devices had slightly positive TCR. GNP/MWNT composites had negative TCR (Figure 5). GNP/MWNT CPDMS that were first annealed $\left(90^{\circ} \mathrm{C}\right)$ exhibited increased TCR (data not shown).

The gauge factors of the devices were calculated from resistance data obtained under known applied strains up to $40 \%$ strain (Figure 6). Gauge factor (along with variability of gauge factor) was observed to increase as the amount of filler material was reduced (Table 1, Figure 7). This is consistent with results reported elsewhere (Table 2) [5-6, 8]. Gauge factor was inversely related to conductivity. Interestingly, gauge factor for sensors with filler content $>12.5 \mathrm{wt} \%$ was increased for CPDMS sesors with Parylene coating. $1.5 \% \quad \mathrm{GNP} / 3.5 \quad \% \mathrm{MWNT}$ composite CPDMS sensors had similar TCR and gauge factor as the pure 5\% MWNT device but had greater conductivity.

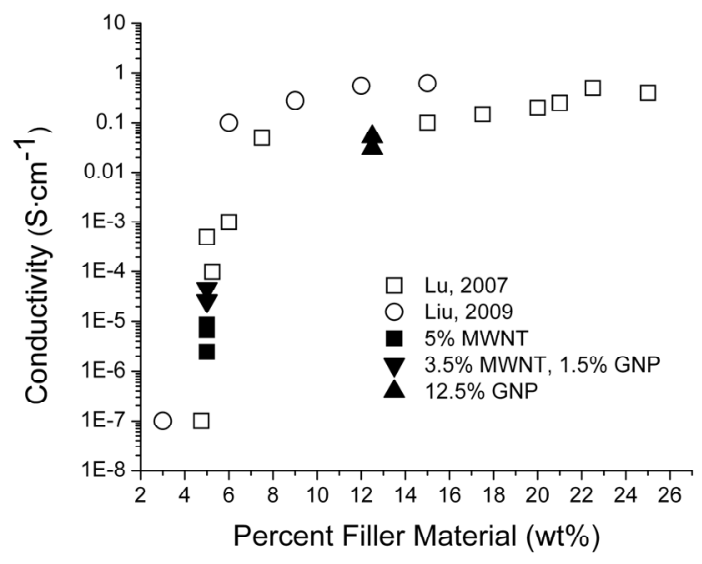

Figure 3: Conductivity measurements for CPDMS samples compared to previously reported data.

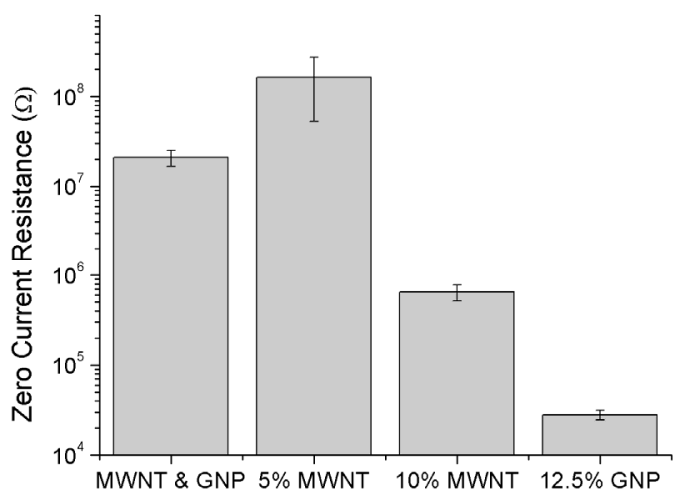

Figure 4: Zero current resistance data (In the composite sample, MWNT \& GNP concentrations are $3.5 \%$ and $1.5 \%$ respectively.). Data are mean $\pm S E(n=6-12)$.

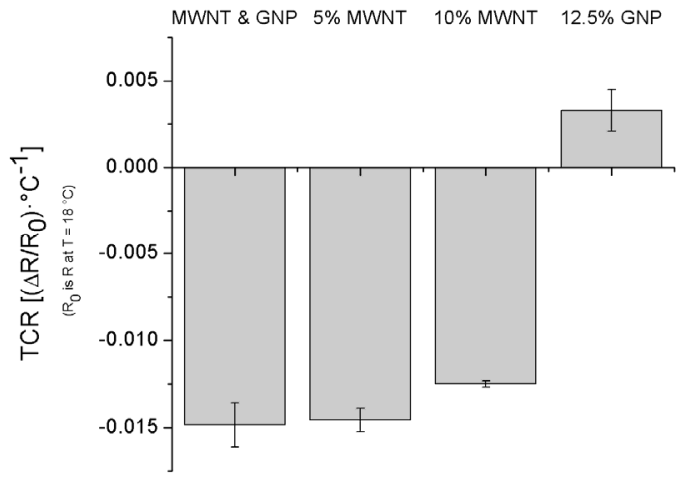

Figure 5: Temperature coefficient of resistance (TCR) data (In the composite sample, MWNT \& GNP concentrations are $3.5 \%$ and $1.5 \%$ respectively.). Data are mean $\pm S E(n=3-5)$. 


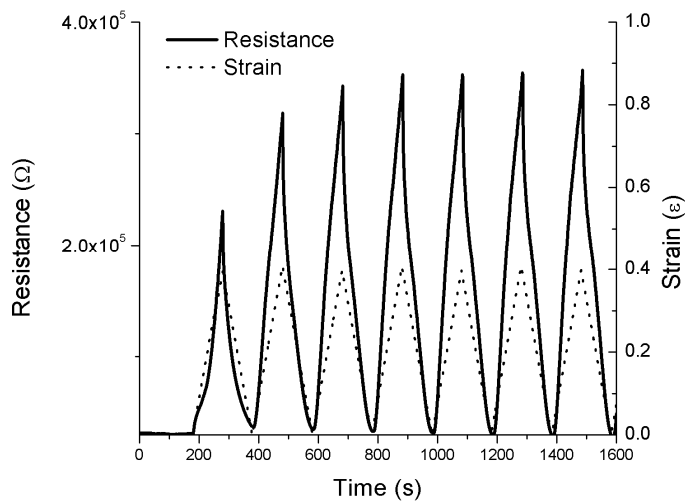

Figure 6: Representative raw gauge factor data. In this case, $40 \%$ strain was applied.

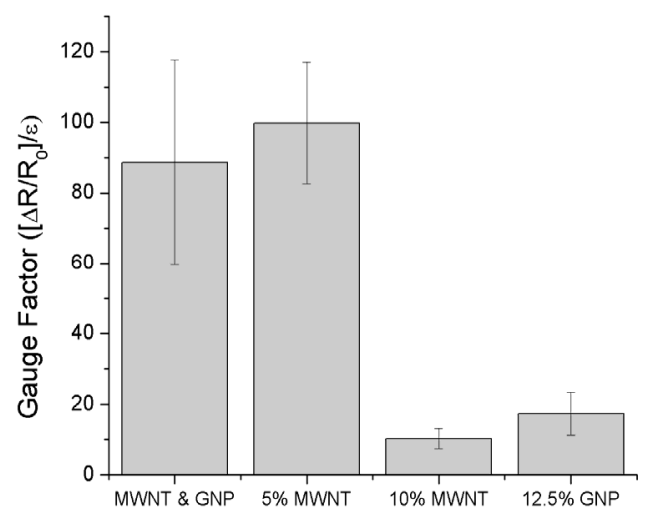

Figure 7: Calculated gauge factor for CDPMS strain sensors having various concentrations and compositions of filler material. Data are mean $\pm S E(n=4-5)$.

Table 2: Gauge factors and achieved strain for CPDMS strain sensors reported in literature.

\begin{tabular}{|c|c|c|}
\hline Reference & Max GF & Max Applied Strain \\
\hline Lu, 2007 [5] & 12.3 & $1.2 \%$ \\
\hline Liu, 2009 [6] & 2.75 & $40 \%$ \\
\hline Giannone, 2009 [8] & 5.6 & $8.5 \%$ \\
\hline
\end{tabular}

Soak testing of strain sensors was performed in $1 \mathrm{X}$ PBS $\left(37^{\circ} \mathrm{C}\right.$ for 21 days) and is ongoing. A decrease in resistance of $42 \%$ over this period was observed, pointing to penetration of conductive ions into the polymer, which can be mitigated by Parylene coating.

\section{CONCLUSION}

Strain sensors made from MWNT and GNP filled PDMS screen-printed onto PDMS were manufactured and characterized according to their zero current resistance, TCR and gauge factor. Due to their high gauge factor, large strain capability, and construction from medical grade materials, these devices are promising for in vivo applications. Coupled with a micro-hydrostatic pressure monitor, these devices could be used to measure bladder fullness for the thousands of people suffering from loss of control of micturition from spinal cord injuries and neurogenic bladder.

\section{ACKNOWLEDGEMENTS}

This work was funded in part by an NSF CAREER Award (ECS-0547544). The authors would like to thank Dr. Donghai Zhu, Mr. Eric Welder, and the members of the USC Biomedical Microsystems Laboratory for their assistance.

\section{REFERENCES}

[1] G. T. A. Kovacs, Micromachined Transducers Sourcebook. McGaw-Hill Companies, Inc. (1998).

[2] M. Madou, Fundamentals of Microfabrication. CRC Press (1997).

[3] G. R. Higson, "Recent advances in strain gauges", Journal of Scientific Instrumentation, 41, 405-414 (1964).

[4] J. Engel, J. Chen, N. Chen, S. Pandya, and C. Liu, "Multiwalled carbon nanotube filled conductive elastomers: materials and application to micro transducers", 19th IEEE International Conference on MEMS, Istanbul, Turkey, 1/22-26/2006, IEEE (2006), pp. 246-249

[5] J. Lu, M. Lu, A. Bermak, Y. Lee, "Study of piezoresistance effect of carbon nanotube-PDMS composite materials for nanosensors", 7th IEEE Conference on Nanotechnology, Hong Kong, China, 8/25/2007, IEEE-NANO (2007) pp. 1240-1243.

[6] C.-X. Liu and J.-W. Choi, "Patterning conductive PDMS nanocomposite in an elastomer using microcontact printing", J. Micromech. and Microeng., 19, 085019 (2009).

[7] I. Kang, et al., "A carbon nanotube strain sensor for structural health monitoring", Smart Materials and Structures, 15, 737-748 (2006).

[8] P. Giannone and S. Graziani, "Flexible tailored sensors for large deformation monitoring", Instrumentation and Measurement Technology Conference, Singapore, 5/57/09, IEEE (2009) pp. 593-596.

[9] R. Rizvi, B.Cochrane, E. Biddiss and H. Naguib "Piezoresistance characterization of poly(dimethylsiloxane) and poly(ethylene) carbon nanotube composites", Smart Mater. Struct., 20, 094003, (2011).

[10] M. Moniruzzaman and K. I. Winey, "Polymer nanocomposites containing carbon nanotubes", Macromolecules, 39, 5194-5205 (2006).

[11] C. A. Gutierrez and E. Meng, "Low-cost carbon thick-film strain sensors for implantable applications", J. Micromech. Microeng., 20, 095028 (2010).

[12] J. E. Brignell, N. M. White, and A. W. J. Cranny, "Sensor applications of thick-film technology", IEE Proc. 135, 77$84(1988)$

[13] J. S. Shah, "Strain sensitivity in thick-film resistors", IEEE Trans. Compon., Hybrids, Manuf. Technol. 3, 554-64 (1980).

[14] M. Prudenziati and B. Morten, "Piezoresistive properties of thick-film resistors: an overview", Microelectronics International, 3, 20-37 (1986).

\section{CONTACT}

*E. Meng., tel: (213) 821 3949; ellis.meng@usc.edu 